\title{
APPLICATION OF THE METHOD OF THE TWO-SIDED APPROXIMATIONS TO THE SOLUTION OF THE PERIODIC PROBLEM FOR IMPULSIVE DIFIERENTIAL EQUATIONS
}

\author{
P. S. SIMEONOV AND D. D. BAINOV
}

\begin{abstract}
In the paper the application of the method of the two-sided approximations to finding the periodic solutions of impulsive diflerential equations is justified.
\end{abstract}

\section{Introduction}

Consider the impulsive $T$-periodic differential equation

$$
\begin{aligned}
& \frac{d x}{d t}=f(t, x), \quad t \neq \tau_{k}, \\
& \Delta x=I_{k}(x), \quad t=\tau_{k},
\end{aligned}
$$

where $t \in \mathbb{R}=(-\infty, \infty), k \in \mathbb{Z}=\{0, \pm 1, \pm 2, \cdots\}, x=\operatorname{col}\left(x_{1}, \ldots, x_{n}\right) \in D \subset \Re^{n}$ and $\mathfrak{R}^{n}$ is an $n$-dimensional vector space with norm $\|x\|=\max _{1 \leq i \leq n}\left|x_{i}\right|$.

Impulsive differential equations of the form (1) are an object of active research in the recent years [1]-[10]. We shall note that the solution $x(t)$ of $(1)$ for $t \neq \tau_{k}$ satisfies the differential equation $d x / d t=f(t, x)$ and for $t=\tau_{k}$ it satisfies the condition for a jump $\Delta x=I_{k}(x)$ and $x\left(\tau_{k}^{+}\right)=x\left(\tau_{k}\right)+I_{k}\left(x\left(\tau_{k}\right)\right), x\left(\tau_{k}^{-}\right)=x\left(\tau_{k}\right)$. Hiere $x\left(\tau_{k}^{ \pm}\right)=$ $\lim _{t \rightarrow \tau_{k} \pm 0} x(t)$.

To find the $T$ periodic solutions of equation (1) we shall apply the method of the two-sided approximations [11].

\section{Preliminary Notes}

Let the functions $g(t, x, y)$ and $J_{k}(x, y)$ be such that

$$
g(t, x, x)=f(t, x), J_{k}(x, x)=I_{k}(x) \quad(t \in \mathbb{R}, k \in \mathbb{Z}, x \in D)
$$

Received November 10, 1990.

The present investigation is supported by the Ministry of Culture,

Science and Education of People's Republic of Bulgaria under Grant 61. 
and instead of (1) consider the equation

$$
\begin{aligned}
\frac{d x}{d t} & =g(t, x, x), & t & \neq \tau_{k}, \\
\Delta x & =J_{k}(x, x), & t & =\tau_{k} .
\end{aligned}
$$

Assume that the following conditions (H) hold:

H1. $\tau_{0}=0, \tau_{k}<\tau_{k+1}(k \in \mathbb{Z})$ and there exists an integer $q>0$ such that $\tau_{k+q}=\tau_{k}+T(k \in \mathbb{Z})$.

H2. The function $g: \mathbb{R} \times D \times D \rightarrow \mathbb{R}^{n}$ is continuous in the sets $\left(\tau_{k-1}, \tau_{k}\right] \times D \times$ $D(k \in \mathbb{Z})$ and for any $x, y \in D$ and $k \in \mathbb{Z}$ there exists the finite limit of $g(t, u, v)$ as $(t, u, v) \rightarrow\left(\tau_{k}, x, y\right), t>\tau_{k}$.

H3. The functions $J_{k}: D \times D \rightarrow \mathbb{R}^{n}(k=1, \cdots, q)$ are continous in $D \times D$.

H4. $g(t+T, x, y)=g(t, x, y)$ and $J_{k+q}(x, y)=J_{k}(x, y)$ for $t \in \mathbb{R}, k \in \mathbb{Z} ; x, y \in$ D.

H5. There exist $M, \mu, L_{k}, \ell_{k} \in \mathbb{R}^{n}$ such that the following inequalities hold

$$
\begin{aligned}
\mu \leq g(t, x, y) & \leq M, \quad \ell_{k} \leq J_{k}(x, y) \leq L_{k} \\
g(t, x, y) & \leq g(t, u, v), \quad J_{k}(x, y) \leq J_{k}(u, v)
\end{aligned}
$$

for $t \in \mathbb{R} ; k \in \mathbb{Z} ; x, y, u, v \in \mathbb{R}^{n}, x \leq u, v \leq y$, where $x \leq u$ means that $x_{i} \leq$ $u_{i}(i=1, \cdots, n)$.

H6. $D=\left\{x \in \mathbb{R}^{n}: a \leq x \leq b\right\}$ and $b-a>\frac{T}{2}(M-\mu)+2 \sum_{k=1}^{q} \max \left(\left|L_{k}\right|,\left|\ell_{k}\right|\right)=2 \epsilon$ where $|x|=\operatorname{col}\left(\left|x_{1}\right|, \ldots,\left|x_{n}\right|\right), \max (x, y)=\operatorname{col}\left(\max \left(x_{1}, y_{1}\right), \ldots, \max \left(x_{n}, y_{n}\right)\right)$.

Remark 1. We shall note that if $N$ is a nonnegative $(n \times n)$-matrix and $-N \leq$ $\frac{\partial f}{\partial x}(t, x) \leq N$ then the function $g(t, x, y)=\frac{1}{2}[f(t, x)+N x]+\frac{1}{2}[f(t, y)-N y]$ satisfies conditions (2) and (5).

If the function $h(t, x)$ is such that

$$
h(t, x)-h(t, u) \leq f(t, x)-f(t, u) \leq-h(t, x)+h(t, u)
$$

for $x \leq u$, then the function

$$
g(t, x, y)=\frac{1}{2}[f(t, x)+h(t, x)]+\frac{1}{2}[f(t, y)-h(t, y)]
$$

also satisfies conditions (2) and (5).

Let $x_{0} \in D_{\epsilon}=\left\{x \in \mathbb{R}^{n}: a+\epsilon \leq x \leq b-\epsilon\right\}$ and define successively the sequences $\left\{u m_{m}\left(t, x_{0}\right)\right\}$ and $\left\{v_{m}\left(t, x_{0}\right)\right\}$ of $T$-periodic functions which in the interval $[0, T]$ 
are given by the formulae:

$$
\begin{aligned}
u_{0}\left(t, x_{0}\right)= & x_{0}-\frac{M-\mu}{2} \alpha(t)+\left(1-\frac{t}{T}\right) \sum_{0 \leq \tau_{k}<t} \ell_{k}-\frac{t}{T} \sum_{t \leq \tau_{k}<T} L_{k} \\
v_{0}\left(t, x_{0}\right)= & x_{0}+\frac{M-\mu}{2} \alpha(t)+\left(1-\frac{t}{T}\right) \sum_{0 \leq \tau_{k}<t} L_{k}-\frac{t}{T} \sum_{t \leq \tau_{k}<T} \ell_{k} \\
u_{m+1}\left(t, x_{0}\right)= & x_{0}+\left(1-\frac{t}{T}\right) \int_{0}^{t} g\left(s, u_{m}\left(s, x_{0}\right), v_{m}\left(s, x_{0}\right)\right) d s \\
& -\frac{t}{T} \int_{t}^{T} g\left(s, v_{m}\left(s, x_{0}\right), u_{m}\left(s, x_{0}\right)\right) d s+\left(1-\frac{t}{T}\right) \sum_{0 \leq \tau_{k}<t} J_{k}\left(u_{m}\left(\tau_{k}, x_{0}\right), v_{m}\left(\tau_{k}, x_{0}\right)\right) \\
& -\frac{t}{T} \sum_{t \leq \tau_{k}<T} J_{k}\left(v_{m}\left(\tau_{k}, x_{0}\right), u_{m}\left(\tau_{k}, x_{0}\right)\right), \\
& -\frac{t}{T} \int_{t}^{T} g\left(s, u_{m}\left(s, x_{0}\right), v_{m}\left(s, x_{0}\right)\right) d s+\left(1-\frac{t}{T}\right) \sum_{0 \leq \tau_{k}<i} J_{k}\left(v_{m}\left(\tau_{k}, x_{0}\right), u_{m}\left(\tau_{k}, x_{0}\right)\right) \\
& -\frac{t}{T} \sum_{t \leq \tau_{k}<T} J_{k}\left(t, u_{m}\left(\tau_{k}, x_{0}\right), v_{m}\left(\tau_{k}, x_{0}\right)\right),
\end{aligned}
$$

where $\alpha(t)=2 t\left(1-\frac{t}{T}\right)$ for $t \in[0, T]$.

We shall find sufficient conditions under which the sequences $u_{m}\left(t, x_{0}\right)$ and $v_{m}\left(t, x_{0}\right)$ two-sided and monotonely tend to the $T$-periodic solution $\tilde{\mathrm{x}}\left(t, x_{0}\right)$ of equation (3) for which $\tilde{x}\left(0, x_{0}\right)=x_{0}$.

In the proof of the main results we shall use the following rclations which are valid for $t \in[G, T]$ :

$$
\begin{gathered}
\left(1-\frac{t}{T}\right) \int_{0}^{t} d s+\frac{t}{T} \int_{t}^{T} d s=\alpha(t) \leq \frac{T}{2} \\
\left(1-\frac{t}{T}\right) \int_{0}^{t} \alpha(s) d s+\frac{t}{T} \int_{t}^{T} \alpha(s) d s=\frac{\alpha^{2}(t)}{3}+\frac{T \alpha(t)}{2} \leq \frac{T}{3} \alpha(t), \\
\left(1-\frac{t}{T}\right) i[0, t)+\frac{t}{T} i[t, T) \leq \eta\left[1-(q-1) \frac{0}{T}\right] \equiv Q, \\
\left(1-\frac{t}{T}\right) \sum_{0 \leq \tau_{k}<t} \alpha\left(\tau_{k}\right)+\frac{t}{T} \sum_{t \leq \tau_{k}<T} \alpha\left(\tau_{k}\right) \leq \frac{8 T q}{27} \equiv S, \\
\left(1-\frac{t}{T}\right) \sum_{0 \leq \tau_{k}<t} \gamma_{k}+\frac{t}{T} \sum_{t \leq \tau_{k}<T} \gamma_{k} \leq \sum_{k=1}^{q} \gamma_{k},
\end{gathered}
$$

where $\gamma_{k} \in \mathbb{R}^{n}, \gamma_{k} \geq 0, i[t, s)$ is the number of point $\left\{\tau_{k}\right\}$ lying in the interval $[t, s)$ and $0=\min _{k=1, \cdots, y}\left(\tau_{k}-\tau_{k-1}\right)$. 


\section{Main Results}

Theorem 1. Let conditions $(H)$ and $x_{0} \in D_{\epsilon}$.

Then:

1) The functions $u_{m}\left(t, x_{0}\right), v_{m}\left(t, x_{0}\right)$ satisfy the relations:

$$
\begin{aligned}
u_{m}\left(0, x_{0}\right) & =u_{m}\left(T, x_{0}\right)=v_{m}\left(0, x_{0}\right)=v_{m}\left(T, x_{0}\right)=x_{0} \\
u_{0}\left(t, x_{0}\right) & \leq u_{1}\left(t, x_{0}\right) \leq \cdots \leq u_{m}\left(t, x_{0}\right) \\
v_{0}\left(t, x_{0}\right) & \geq v_{1}\left(t, x_{0}\right) \geq \cdots \geq v_{m}\left(t, x_{0}\right) \\
a & \leq u_{m}\left(t, x_{0}\right) \leq v_{m}\left(t, x_{0}\right) \leq b
\end{aligned}
$$

for $t \in[0, T], m=0,1,2, \cdots$.

2) The sequences $\left\{u_{m}\left(t, x_{0}\right)\right\},\left\{v_{m}\left(t, x_{0}\right)\right\}$ are uniformly convergent in the interval $[0, T]$ and their limits $u\left(t, x_{0}\right), v\left(t, x_{0}\right)$ satisfy the relations:

$$
\begin{aligned}
u\left(0, x_{0}\right)= & u\left(T, x_{0}\right)=v\left(0, x_{0}\right)=v\left(T, x_{0}\right)=x_{0}, \\
u_{m}\left(t, x_{0}\right) \leq & u\left(t, x_{0}\right) \leq v\left(t, x_{0}\right) \leq v_{m}\left(t, x_{0}\right)(t \in[0, T], m=0,1,2, \cdots), \\
u\left(t, x_{0}\right)= & x_{0}+\left(1-\frac{t}{T}\right) \int_{0}^{t} g\left(s, u\left(s, x_{0}\right), v\left(s, x_{0}\right)\right) d s \\
& -\frac{t}{T} \int_{i}^{T} g\left(s, v\left(s, x_{0}\right), u\left(s, x_{0}\right)\right) d s+\left(1-\frac{t}{T}\right) \sum_{0 \leq \tau_{k}<t} J_{k}\left(u\left(\tau_{k}, x_{0}\right), v\left(\tau_{k}, x_{0}\right)\right) \\
& -\frac{t}{T} \sum_{t \leq T_{k}<T} J_{k}\left(v\left(\tau_{k}, x_{0}\right), u\left(\tau_{k}, x_{0}\right)\right), \\
v\left(t, x_{0}\right)= & x_{0}+\left(1-\frac{t}{T}\right) \int_{0}^{t} g\left(s, v\left(s, x_{0}\right), u\left(s, x_{0}\right)\right) d s \\
& -\frac{t}{T} \int_{i}^{T} g\left(s, u\left(s, x_{0}\right), v\left(s, x_{0}\right)\right) d s+\left(1-\frac{t}{T}\right) \sum_{0 \leq \tau_{k}<i} J_{k}\left(v\left(\tau_{k}, x_{0}\right), u\left(\tau_{k}, x_{0}\right)\right) \\
& -\frac{t}{T} \sum_{t \leq \tau_{k}<T} J_{k}\left(u\left(\tau_{k}, x_{0}\right), v\left(\tau_{k}, x_{0}\right)\right) .
\end{aligned}
$$

\section{Proof.} that

1) The validity of (15) is obvious. From (6), (7), HG and property (14) it follows

$$
a \leq u_{0}\left(t, x_{0}\right) \leq v_{0}\left(t, x_{0}\right) \leq b .
$$

Taking into account (4) and (9), we obtain that

$$
v_{1}\left(t, x_{0}\right) \leq x_{0}+\left(1-\frac{t}{T}\right) \int_{0}^{\imath} M d s-\frac{t}{T} \int_{t}^{T} \mu d s+\left(1-\frac{t}{T}\right) \sum_{0 \leq \tau_{k}<\imath} L_{k}-\frac{t}{T} \sum_{t \leq \tau_{k}<T} \ell_{k}=v_{0}(t, x)
$$


for $t \in[0, T]$. Analogously, $u_{0}\left(t, x_{0}\right) \leq u_{1}\left(t, x_{0}\right)$ for $t \in\left[0, T_{j}\right.$.

From (8), (9), (23) and condition (5) it follows that

$$
\begin{aligned}
& v_{1}\left(t, x_{0}\right)-u_{1}\left(t, x_{0}\right) \\
= & \left(1-\frac{t}{T}\right) \int_{0}^{t}\left[g\left(s, v_{0}\left(s, x_{0}\right), u_{0}\left(s, x_{0}\right)\right)-g\left(s, u_{0}\left(s, x_{0}\right), v_{0}\left(s, x_{0}\right)\right)\right] d s \\
& +\frac{t}{T} \int_{t}^{T}\left[g\left(s, v_{0}\left(s, x_{0}\right), u_{0}\left(s, x_{0}\right)\right)-g\left(s, u_{0}\left(s, x_{0}\right), v_{0}\left(s, x_{0}\right)\right)\right] d s \\
& +\left(1-\frac{t}{T}\right) \sum_{0 \leq \tau_{k}<\imath}\left[J_{k}\left(v_{0}\left(\tau_{k}, x_{0}\right), u_{0}\left(\tau_{k}, x_{0}\right)\right)-J_{k}\left(u_{0}\left(\tau_{k}, x_{0}\right), v_{0}\left(\tau_{k}, x_{0}\right)\right)\right] \\
& +\frac{t}{T} \sum_{t \leq T_{k}<T}\left[J_{k}\left(v_{0}\left(\tau_{k}, x_{0}\right), u_{0}\left(\tau_{k}, x_{0}\right)\right)-J_{k}\left(u_{0}\left(\tau_{k}, x_{0}\right), v_{0}\left(\tau_{k}, x_{0}\right)\right)\right]
\end{aligned}
$$

Thus for $t \in[0, T]$ we have

$$
a \leq u_{0}\left(t, x_{0}\right) \leq u_{1}\left(t, x_{0}\right) \leq v_{1}\left(t, x_{0}\right) \leq v_{0}\left(t, x_{0}\right) \leq b
$$

By induction on $m$ in virtue of (24) it is proved that for any $m=0,1,2, \cdots$ and $t \in[0, T]$

$$
u_{m+1}\left(t, x_{0}\right) \leq u_{m}\left(t, x_{0}\right) \leq v_{m}\left(t, x_{0}\right) \leq v_{m+1}\left(t, x_{0}\right)
$$

2) Consider the space $P C\left(\mathbb{R}, \mathbb{R}^{n}\right)$ of piecewise continuous functions $x: \mathbb{R} \rightarrow \mathbb{R}^{n}$ which have points of discontinuity $\tau_{k}(k \in \mathbb{Z})$ and are continuous from the left in $\mathbb{R}$. Let the norm of $x \in P C\left(\mathbb{R}, \mathbb{R}^{n}\right)$ be $\|x\|_{P C}=\sup _{t \in \mathbb{R}}\|x(t)\|$.

From conditions $\mathrm{H} 2$ and $\mathrm{H} 3$ it follows that the functions $u_{m}\left(t, x_{0}\right)$ and $v_{m}\left(t, x_{0}\right)$ belong to $P C\left(\mathbb{R}, \mathbb{R}^{n}\right)$. Since the sequences $\left\{u_{m}\left(t, x_{0}\right)\right\}$ and $\left\{v_{m}\left(t, x_{0}\right)\right\}$ are uniformly bounded and quasiequicontinuous [5], then by Lemma 4, [5] they have convergent subsequences. But from the monotonicity of the sequences $\left\{u_{m}\left(t, x_{0}\right)\right\}$ and $\left\{v_{m}\left(t, x_{0}\right)\right\}$ it follows that $u_{m}\left(t, x_{0}\right)$ and $v_{m}\left(t, x_{0}\right)$ are convergent in $P C\left(\mathbb{R}, \mathbb{R}^{n}\right)$ which means that there exist functions $u\left(t, x_{0}\right)$ and $v\left(t, x_{0}\right)$ of $P C\left(\mathbb{R}, \mathbb{R}^{n}\right)$ for which

$$
\lim _{m \rightarrow \infty} u_{m}\left(t, x_{0}\right)=u\left(t, x_{0}\right), \quad \lim _{m \rightarrow \infty} v_{m}\left(t, x_{0}\right)=v\left(t, x_{0}\right)
$$

uniformly with respect to $t \in[0, T]$.

(15)-(18) and (25) imply immediately the validity of relations (19)-(22). Theorem 1 is proved.

Consider the equation

$$
\begin{aligned}
x(t)= & x_{0}+\left(1-\frac{t}{T}\right) \int_{0}^{t} g(s, x(s), x(s)) d s-\frac{t}{T} \int_{t}^{T} g(s, x(s), x(s)) d s \\
& +\left(1-\frac{t}{T}\right) \sum_{0 \leq \tau_{k}<t} J_{k}\left(x\left(\tau_{k}\right), x\left(\tau_{k}\right)\right)-\frac{t}{T} \sum_{t \leq \tau_{k}<T} J_{k}\left(x\left(\tau_{k}\right), x\left(\tau_{k}\right)\right) .
\end{aligned}
$$


Theorem 2. Let conditions (H) hold and $x_{0} \in D_{\epsilon}$.

Then equation (26) has a $T$-periodic solution $x^{*}(t)$ and the following relations hold:

$$
\begin{array}{ll}
x^{*}(0)=x^{*}(T)=x_{0}, & \\
u_{m}\left(t, x_{0}\right) \leq x^{*}(t) \leq v_{m}\left(t, x_{0}\right) & (t \in[0, T], m=0,1,2, \cdots), \\
u\left(t, x_{0}\right) \leq x^{*}(t) \leq v\left(t, x_{0}\right) & (t \in[0, T]) .
\end{array}
$$

Proof. Consider the set $\Omega$ of functions $x \in P C\left(\mathbb{R}, \mathbb{R}^{n}\right)$ which are $T$-periodic and satisfy the conditions

$$
x(0)=x(T)=x_{0}, \quad a \leq x(t) \leq b .
$$

Define the operator $\mathcal{F}: \Omega \rightarrow P C\left(\mathbb{R}, \mathbb{R}^{n}\right)$ by the formula

$$
\begin{aligned}
\mathcal{F}_{x(t)}= & x_{0}+\left(1-\frac{t}{T}\right) \int_{0}^{t} g(s, x(s), x(s)) d s-\frac{t}{T} \int_{i}^{T} g(s, x(s), x(s)) d s \\
& +\left(\mathbb{1}-\frac{t}{T}\right) \sum_{0 \leq \tau_{k}<t} J_{k}\left(x\left(\tau_{k}\right), x\left(\tau_{k}\right)\right)-\frac{t}{T} \sum_{i \leq \tau_{k}<T} J_{k}\left(x\left(\tau_{k}\right), x\left(\tau_{k}\right)\right) .
\end{aligned}
$$

The following assertions are valid:

I. The set $\Omega$ is bounded, convex and closed in $P C\left(\mathbb{R}, \mathbb{R}^{n}\right)$.

II. $\mathcal{F}$ maps $\Omega$ into itself. Indeed, if $x \in \Omega$, then

$$
\begin{aligned}
\mathcal{F}_{x(t) \leq} \leq x_{0}+\left(1-\frac{t}{T}\right) \int_{0}^{t} M d s-\frac{t}{T} \int_{i}^{T} \mu d s \\
\quad+\left(1-\frac{t}{T}\right) \sum_{0 \leq \tau_{k}<t} L_{k}-\frac{t}{T} \sum_{t \leq T_{k}<t} \ell_{k}=v_{0}\left(t, x_{0}\right) \leq b .
\end{aligned}
$$

Analogously,

$$
\mathcal{F}_{x(t)} \geq u_{0}\left(t, x_{0}\right) \geq a
$$

and since $\mathcal{F}_{x}(0)=\mathcal{F}_{x}(T)=x_{0}$ and $\mathcal{F}_{x} \in P C\left(\mathbb{R}, \mathbb{R}^{n}\right)$, then $\mathcal{F}_{x} \in \Omega$.

III. The set $\mathcal{F} \Omega$ is relatively compact in $P C\left(\mathbb{R}, \mathbb{R}^{n}\right)$. For the proof of this assertion we apply Lemma 4 , [5], taking into account that $\mathcal{F} \Omega$ is uniformly bounded and quasiequicontinuous. We shall mention only that the quasiequicontinuity of $\mathcal{F} \Omega$ follows from $\mathbb{H} 2, \mathbb{H} 3$ and the equality

$$
\begin{aligned}
F_{x}\left(t_{2}\right)-\mathcal{F}_{x}\left(t_{1}\right)= & \int_{\imath_{1}}^{t_{2}} g(s, x(s), x(s)) d s \\
& +\frac{t_{1}-t_{2}}{T}\left[\int_{0}^{T} g(s, x(s), x(s)) d s+\sum_{k=1}^{q} J_{k}\left(x\left(\tau_{k}\right), x\left(\tau_{k}\right)\right)\right]
\end{aligned}
$$


for $x \in \Omega$ and $t_{1}, t_{2} \in\left(\tau_{k-1}, \tau_{k}\right], t_{1}<t_{2}(k \in \mathbb{Z})$.

Hence by the Schauder-Tychonoff theorem the operator $\mathcal{F}$ has a fixed point $x^{*} \in \Omega$, i.e. there exists a $T$-periodic function $x^{*}(t)$ satisfying (26). From (29) and $(30)$ there follows the estimate

$$
u_{0}\left(t, x_{0}\right) \leq x^{*}(t) \leq v_{0}\left(t, x_{0}\right)
$$

from which by induction on $m=0,1,2, \cdots$ we obtain that

$$
u_{m}\left(t, x_{0}\right) \leq x^{*}(t) \leq v_{m}\left(t, x_{0}\right) \quad(t \in[0, T], m=0,1,2, \cdots) .
$$

In (31) we pass to the limit and obtain (28).

Theorem 3. Let the following conditions be fulfilled:

1) Conditions (H) hold and $x_{0} \in D_{\epsilon}$.

2) The functions $g$ and $J_{k}$ satisfy the estimates

$$
\begin{aligned}
g(t, x, y)-g(t, y, x) & \leq K(x-y) \\
J_{k}(x, y)-J_{k}(y, x) & \leq C(x-y)
\end{aligned}
$$

where $a \leq y \leq x \leq b$ and $K$ and $C$ are nonnegative $(n \times n)$-matrices.

3) The modules of the eigenvalues of the matrix

$$
P=\left[\begin{array}{cc}
\frac{T}{3} K & K \\
S C & Q C
\end{array}\right]
$$

are less than 1 .

Then equation (26) has a unique $T$-periodic solution $\tilde{x}\left(t, x_{0}\right)$ for which $\tilde{x}\left(0, x_{0}\right)=x_{0}$ and

$$
\widetilde{x}\left(t, x_{0}\right)=u\left(t, x_{0}\right)=v\left(t, x_{0}\right) \quad(t \in[0, T]) .
$$

Proof. For $m=0$ the following estimate is valid

$$
\begin{aligned}
v_{0}\left(t, x_{0}\right)-u_{0}\left(t, x_{0}\right) & =\alpha(t)(M-\mu)+\left(1-\frac{t}{T}\right) \sum_{0 \leq \tau_{k}<\ell}\left(L_{k}-\ell_{k}\right)+\frac{t}{T} \sum_{t \leq \tau_{k}<T}\left(L_{k}-\ell_{k}\right) \\
& \leq \alpha(t)(M-\mu)+\sum_{k=1}^{q}\left(L_{k}-\ell_{k}\right)=\alpha(t) a_{0}+b_{0} .
\end{aligned}
$$

For $m=j$ let us have that

$$
v_{j}\left(t, x_{0}\right)-u_{j}\left(t, x_{0}\right) \leq \alpha(t) a_{j}+b_{j}
$$


Then by (8), (9), (32), (10)-(13) we obtain

$$
\begin{aligned}
& v_{j+1}\left(t, x_{0}\right)-u_{j+1}\left(t, x_{0}\right) \\
\leq & \left(1-\frac{t}{T}\right) \int_{0}^{t} K\left[\alpha(s) a_{j}+b_{j}\right] d s+\frac{t}{T} \int_{t}^{T} K\left[\alpha(s) a_{j}+b_{j}\right] d s \\
& +\left(1-\frac{t}{T}\right) \sum_{0 \leq \tau_{k}<t} C\left[\alpha\left(\tau_{k}\right) a_{j}+b_{j}\right]+\frac{t}{T} \sum_{t \leq \tau_{k}<T} C\left[\alpha\left(\tau_{k}\right) a_{j}+b_{j}\right] \\
\leq & \alpha(t)\left[\frac{T}{3} K a_{j}+K b_{j}\right]+\left[S C a_{j}+Q C b_{j}\right] .
\end{aligned}
$$

Consequently, for $m=0,1,2, \cdots$ the following estimates are valid

$$
v_{m 3}\left(t, x_{0}\right)-\imath_{m}\left(t, x_{0}\right) \leq \alpha(t) a_{m}+b_{m} \quad(t \in[0, T])
$$

where

$$
\begin{array}{ll}
a_{m+1}=\frac{T}{3} K a_{m}+K b_{m}, & a_{0}=M-\mu, \\
b_{m+1}=S C a_{m}+Q C b_{m}, & b_{u}=\sum_{k=1}^{q}\left(L_{k}-\ell_{k}\right) .
\end{array}
$$

From (3), in view of condition 3 it follows that $a_{m} \rightarrow 0$ and $b_{m} \rightarrow 0$ as $m \rightarrow \infty$, i.e.

$\lim _{m \rightarrow \infty}\left[v_{m}\left(t, x_{0}\right)-u_{m}\left(t, x_{0}\right)\right]=0 \quad$ (uniformly with respect to $t \in[0, T]$ ).

Then $u\left(t, x_{0}\right)=v\left(t, x_{0}\right)$ and by Theorem 2

$$
\widetilde{x}\left(t, x_{0}\right)=x^{*}(t)=u\left(t, x_{0}\right)=v\left(t, x_{0}\right) \quad(t \in[0, T]) .
$$

Theorem 3 is proved.

Under the conditions of Theorem 3 we shall consider the question of existence of a $T$-periodic solution of equation (3).

Introduce the mapping $\Delta\left(x_{0}\right): D_{\epsilon} \rightarrow \mathbb{R}^{n}:$

$$
\Delta\left(x_{0}\right)=\frac{1}{T} \int_{0}^{T} g\left(s, \widetilde{x}\left(s, x_{0}\right), \widetilde{x}\left(s, x_{0}\right)\right) d s+\frac{1}{T} \sum_{k=1}^{q} J_{k}\left(\widetilde{x}\left(\tau_{k}, x_{0}\right), \widetilde{x}\left(\tau_{k}, x_{0}\right)\right),
$$

where $\widetilde{x}\left(t, x_{0}\right)$ is the $T$-periodic solution of equation (26) for which $\widetilde{x}\left(0, x_{0}\right)=x_{0}$.

Since

$$
\begin{aligned}
\tilde{x}\left(t, x_{0}\right)= & x_{0}+\int_{0}^{t} g\left(s, \widetilde{x}\left(s, x_{0}\right), \widetilde{x}\left(s, x_{0}\right)\right) d s \\
& +\sum_{0 \leq \tau_{k}<i} J_{k}\left(\widetilde{x}\left(\tau_{k}, x_{0}\right), \widetilde{x}\left(\tau_{k}, x_{0}\right)\right)-t \Delta\left(x_{0}\right)
\end{aligned}
$$


then $\widetilde{x}\left(t, x_{0}\right)$ is a. $T$-periodic solution of $(3)$ if and only if $\Delta\left(x_{0}\right)=0$.

From condition (5) and (27) it follows that

$$
\Delta_{m}\left(x_{0}\right) \leq \Delta\left(x_{0}\right) \leq \Delta^{m}\left(x_{0}\right) \quad\left(x_{0} \in D_{\varepsilon}\right)
$$

where

$$
\begin{aligned}
& \Delta_{m}\left(x_{0}\right)=\frac{1}{T} \int_{0}^{T} g\left(s, u_{m}\left(s, x_{0}\right), v_{m}\left(s, x_{0}\right)\right) d s+\frac{1}{T} \sum_{k=1}^{q} J_{k}\left(u_{m}\left(\tau_{k}, x_{0}\right), v_{m}\left(\tau_{k}, x_{0}\right)\right), \\
& \Delta^{m}\left(x_{0}\right)=\frac{1}{T} \int_{0}^{T} g\left(s, v_{m}\left(s, x_{0}\right), u_{m}\left(s, x_{0}\right)\right) d s+\frac{1}{T} \sum_{k=1}^{q} J_{k}\left(v_{m}\left(\tau_{k}, x_{0}\right), u_{m}\left(\tau_{k}, x_{0}\right)\right) .
\end{aligned}
$$

Inequalities (34) imply the following theorem.

Theorem 4. Let the conditions of Theorem 3 hold and for some integer $m \geq 0$, $\Delta_{m}\left(x_{0}\right)>0$ or $\Delta^{m}\left(x_{0}\right)<0$.

Then equation (3) has no $T$-periodic solution $x(t)$ for which $x(0)=x_{0}$.

The following theorem is also valid.

Theorem 5. Let the following conditions be fulfilled:

1) The conditions of Theorem 3 hold.

2) For some integer $m \geq 0$ the mapping $\triangle_{m}\left(x_{0}\right)$ has an isolated singular point $x_{0}$ $\left(\Delta_{m}\left(x_{0}\right)=0\right)$.

3) The index of the singular point $x_{0}$ is different from zero.

4) There exists a closed domain $F \subset D_{\epsilon}$ with a unique singular point $x_{0}$ such that on its boundary $\partial \mathrm{F}$ the inequality

$$
\left\|\left[\frac{T}{3} K+\frac{1}{T} \sum_{k=1}^{q} a\left(\tau_{k}\right) C\right] a_{m}+\left[K+\frac{q}{T} C\right] b_{m}\right\| \leq \inf _{x \in \partial F}\left\|\Delta_{m}(x)\right\|
$$

holds, where $a_{m}$ and $b_{m}$ are defined by formulae (33).

Then equation (3) has a $T$-periodic solution $2(t)$ for which $x(0) \in \mathbb{F}$.

Proof. Following the prool of Theorem 5. 5, [11], p. 166, it suffices to prove that

$$
\left\|\Delta_{m}(x)\right\|>\left\|\Delta(x)-\Delta_{m}(x)\right\| \quad(x \in \partial \mathbb{F})
$$

which follows from (34), (35) and the estimate

$$
\begin{aligned}
0 & \leq \Delta(x)-\Delta_{m}(x) \leq \Delta^{m}(x)-\Delta_{m}(x) \\
& \leq \frac{1}{T} \int_{0}^{T} K\left(v_{m}\left(s, x_{0}\right)-u_{m}\left(s, x_{0}\right)\right) d s+\frac{1}{T} \sum_{k=1}^{q} C\left(v_{m}\left(\tau_{k}, x_{0}\right)-u_{m}\left(\tau_{k}, x_{0}\right)\right) \\
& \leq \frac{1}{T} \int_{0}^{T} K\left[\alpha(s) a_{m}+b_{m}\right] d s+\frac{1}{T} \sum_{k=1}^{q} C\left[\alpha\left(\tau_{k}\right) a_{m}+b_{m}\right] \\
& =\left[\frac{T}{3} K+\frac{1}{T} \sum_{k=1}^{q} \alpha\left(\tau_{k}\right) C\right] a_{m}+\left[K+\frac{q}{T} C\right] b_{m} .
\end{aligned}
$$




\section{Refelences}

[1] Simeonov P. S., Bainov D. D., "Stability in linear approximation of systems with impulse effect", Rend. Sem. Mat. Univers. Politecn. Torino, Vol. $43^{\circ}, 2,303-322,1985$.

[2] Simeonov P. S., Bainov D. D., "Stability with respect to part of the variables in systems with impulse effect", J. Math. Anal. Appl., Vol. 117, No. 1, 247-263, 1986.

[3] Simeonov P. S., Bainov D. D., "Stability of the solutions of singularly perturbed systems with impulse effect", J. Math. Anal. Appl., Vol. 136, No. 2, 575-588, 1988.

[4] Simeonov P. S., Bainov D. D., "Perturbation theorems for systems with impulse effect", Int. J. Systems Sci., Vol. 19, No. 7, 1213-1223, 1988.

[5] Simeonov P.S., Bainov D. D., "On the asymptotic equivalence of systems with impulse effect", J. Math. Anal. Appl., Vol. 135, No. 2, 591-610, 1988.

[6] Lakshmikantham V., Bainov D. D., Simeonov P. S., "Theory of Impulsive Differential Systems", World Scientific Publishing Co. Pte. Ltd, Singapore-New Jersey-London-Hong Kong.

[7] Bainov D. D., Hu Shouchuan, Hristova S. G., Lakshmikantham V., "Periodic boundary value problems for systems of first order impulsive differential equations", J. Diff. and Integral Equations (to appear).

[8] Lakshmikantham V., Liu Xinzhi., "Stability criteria for impulsive differential equations in terms of two measures", J. Math. Anal. Appl., Vol. 135, 591-604, 1989.

[9] Hu Shouchuan, Lakshmikantham V., Leela S., "Impulsive differential equations and the pulse phenomena", J. Math. Anal. Appl., Vol. 137, 605-612, 1989.

[10] Lakshmikantham V., Liu Xinzhi., "On quasi stability for impulsive differential systems", Nonlinear Analysis (to appear).

[11] Mitropol'skii Yu. A., Martynyuk D. I., "Periodic and quasiperiodic oscillations of systems with delay", "Višča Škola", Kiev, 1979. (in Russian).

Plovdiv University, "Paissij Hilendarski". 